\title{
Original
}

\section{Comparison of CT Urography and MRI in Bladder Cancer Detection}

\author{
Akifumi NirYa, Yoshimitsu OHGIYA, \\ Noritaka SEINO and Takehiko GOKAN*
}

\begin{abstract}
A final diagnosis of bladder cancer is made based on a pathological assessment using cystoscopy and biopsy. Recently, computed tomography (CT) and magnetic resonance imaging (MRI) have become widely used as screening tests for hematuria, and there are scattered reports of new imaging modalities such as CT urography (CTU) and diffusion-weighted MRI being useful in the detection of bladder cancer. However, there are no reports comparing CTU and MRI in this context. In the present study, we compared the bladder cancer detection abilities of CTU and MRI. We analyzed 58 cases of bladder cancer that had been examined by both CTU and bladder MRI. The objects of comparison were T2-weighted images and diffusion-weighted images for MRI and contrast CT images of the renal parenchyma and excretory phases for CTU. Bladder cancer was confirmed histopathologically via either biopsy or surgery for all cases. For patients with multiple bladder cancer, up to three lesions per case were included in the analysis. Two independent readers assessed all cases. Out of 91 lesions from 58 cases, Reader 1 detected $72(79.1 \%)$ and 65 cases (71.4\%), and Reader 2 detected $69(75.8 \%)$ and $70(76.9 \%)$, using MRI and CTU, respectively. The $\kappa$-values for Reader 1 versus Reader 2 were 0.780 for MRI and 0.857 for CT, showing high diagnostic consistency. MRI showed a higher lesion detection rate than CTU, but this difference was not statistically significant. This study showed no significant difference in bladder cancer detection rate between CTU and MRI, confirming the value of MRI in the clinical diagnosis of bladder cancer.
\end{abstract}

Key words : bladder cancer, CT urography, MRI

\section{Introduction}

Bladder cancer is the most frequently occurring malignant tumor of the urinary tract ${ }^{1,2)}$. The most common symptom of bladder cancer is hematuria, and when hematuria is present and bladder cancer is clinically suspected, ultrasonography and cystoscopy are typically performed as screening examinations ${ }^{1,3)}$. Excretory urography is another urinary tract screening method that has long been used, but it has become increasingly replaced by CT urography (CTU) in recent times $^{4-7)}$. CTU involves thin-section imaging of the urinary tract by multidetector CT (MDCT) during the excretory phase after intravenous injection of an iodine contrast agent, at high spatial and contrast resolution. CTU has proven especially useful in the diagnosis of renal pelvic and 
urethral cancers ${ }^{8,9)}$, and in detecting bladder cancer ${ }^{10-12)}$. A patient presenting with macroscopic hematuria is recommended to undergo CTU in combination with cystoscopy and ultrasonography, with a differential diagnosis including urothelial cancer ${ }^{4,13)}$. However, CTU uses contrast agents, meaning patients who suffer from reduced renal function or iodine allergy are precluded, and patients are subject to high X-ray exposure. In conjunction with the development of devices like 3T MRI equipment, new imaging modalities such as diffusion-weighted imaging are now being used in various domains for detecting cancer and determining its spread, with the major advantage that MRI does not involve contrast agents ${ }^{14,15)}$. To our knowledge, here we present the first direct comparison of CTU and MRI for the detection of bladder cancer.

\section{Methods}

All cases for this study were selected from patients undergoing cystoscopy from Jan 2008 to Dec 2014 at Showa University Hospital. Of patients diagnosed with bladder cancer based on biopsy or surgery, we selected 58 who had undergone CTU and bladder MRI examinations within one month of each other. The mean age was 67 years (range : 35-84 years), with 46 males and 12 females. We selected only cases for which a minimum of T2-weighted and diffusion-weighted MRI imaging was implemented. The MRI comprised whole-body 3T MRI (MAGNETOM Trio, A Tim System 3T: Siemens, Germany) and whole-body 1.5T MRI (MAGNETOM Avanto 1.5T: Siemens, Germany; MAGNETOM ESSENZA 1.5T: Siemens, Germany; Singa HDxt 1.5T: G.E., USA), with 3T devices used for 45 cases and $1.5 \mathrm{~T}$ devices for 13 cases. For the 3T MRI devices, T2-weighted images were captured with fast spin-echo imaging under settings of $\mathrm{TR} / \mathrm{TE}=4000-4500 / 82-88 \mathrm{~ms}$ and $\mathrm{FOV}=22 \mathrm{~cm}$; diffusion-weighted images were captured under settings of $\mathrm{TR} / \mathrm{TE}=3300-3800 / 68-70 \mathrm{~ms}$, B-value $=0,50,5000,1000 \mathrm{~s} / \mathrm{mm}$, and FOV $=35-38 \mathrm{~cm}$. For $1.5 \mathrm{~T}$ MRI devices, T2-weighted images were captured with fast spinecho imaging under settings of $\mathrm{TR} / \mathrm{TE}=4000 \sim 4500 / 91 \sim 99 \mathrm{~ms}$ and FOV $=22 \mathrm{~cm}$; diffusionweighted images were captured under settings of $\mathrm{TR} / \mathrm{TE}=3300 \sim 3800 / 77 \sim 84 \mathrm{~ms}$, B-value $=$ $50,5000,1000 \mathrm{~s} / \mathrm{mm}$, and FOV $=35 \mathrm{~cm}$.

The CT procedures comprised 64-slice MDCT (SOMATOM Sensation 64: Siemens, Germany), 128-slice MDCT (SOMATOM Definition AS: Siemens, Germany), and 4-slice MDCT (Light Speed Plus: G.E., USA). After simple CT from the upper pole of the kidney to the bladder, $100 \mathrm{ml}$ of non-ionic iodine contrast agent $(300 \mathrm{mg} / \mathrm{ml})$ was intravenously injected for CTU : after 80 seconds, the renal parenchyma phase was imaged from above the diaphragm to the pubis, and after 8 minutes, the excretory phase was imaged from the upper pole of the kidney to the bladder. If sufficient contrast was not achieved in the 8-min images, those hematuria patients 40 years and older were subjected to a second round of imaging, 12 minutes after the initial injection, from the upper-pole kidney to the bladder. Three-dimensional images were constructed using excretory phase images, including maximum intensity projection (MIP) and volume rendering (VR) images. For simple CT, renal parenchyma phase CT, and excretory phase CT, 5-mm axial images were captured; coronal images were also captured for the excretory phase.

In the evaluation of bladder cancer, up to three lesions per patient were included in analysis 
for cases of multiple bladder cancer. Two independent evaluators-Reader 1 and Reader 2diagnosed the presence of bladder tumors using MRI and CT axial images. T2-weighted and diffusion-weighted images were interpreted and evaluated for MRI, while post-contrast images of the renal parenchyma and excretory phases were examined for CTU. For cases where bladder cancer was diagnosed based on histopathological findings, the diagnosis results for CT and MRI images by Reader 1 and Reader 2 were compared with cystoscopic and surgical findings. Statistical analysis by McNemar's test was conducted using SPSS version 17.0. $P$-values of $<0.05$ were considered statistically significant. Ethical approval for this retrospective study was granted by the institutional review board, and patient consent was not required.

\section{Results}

The observed bladder cancers showed slightly high signals in T2-weighted MRI images and high signals in diffusion-weighted MRI images. In contrast, the, CT images exhibited strongly enhanced signals after the administration of contrast agent for 80 cases; in the excretory phase, contrast defects were observed inside bladders filled with contrast agent (Fig. 1-3). The maximum diameter of tumors detectable via CT or MRI was 2.42-67.2 mm (mean : 19.0 mm); of the 73 detectable lesions, 16 were $\geq 30 \mathrm{~mm}$ in diameter, 14 were $>20 \mathrm{~mm}$ and $\leq 30 \mathrm{~mm}, 19$ were $>10 \mathrm{~mm}$ and $\leq 20 \mathrm{~mm}$, and 24 were $\leq 10 \mathrm{~mm}$. Detected bladder cancers were classified by location according to the publication Cancer Treatment Standards ${ }^{16)}$, and comprised 4 apical lesions, 3 anterior-wall lesions, 13 posterior-wall lesions, 14 trigonal lesions, 29 left-wall lesions, 14 right-wall lesions, 11 bladder-neck lesions, 2 prostatic urethral lesions, 1 trigonal-rear-wall-left-wall lesion, 1 trigonal-right-wall lesion, and 1 left-trigonal-posterior-wall lesion. In terms of T-stage, there were $53 \mathrm{Ta}, 33 \mathrm{~T} 1,4 \mathrm{~T} 3 \mathrm{a}$, and $1 \mathrm{~T} 4 \mathrm{a}$ lesions.

Using both MRI and CTU for 91 lesions from 58 cases, Reader 1 detected 72 (79.1\%) and 65 cases $(71.4 \%)$, respectively, and Reader 2 detected $69(75.8 \%)$ and $70(76.9 \%)$ cases, respectively (Table 1). The $\kappa$-values for Reader 1 versus Reader 2 were 0.780 for MRI and 0.857 for CT, showing high diagnostic consistency. MRI showed a higher lesion detection rate than CTU, but this difference was not statistically significant, with $P$-values of 0.065 for Reader 1 and 1.00 for Reader 2.

\section{Discussion}

One of the characteristic features of bladder carcinoma is the multitude of occurrences in time and space, thus there is a high frequency of recurrence. In this study, there were multiple lesions in 21 cases; however, we evaluated only three lesions maximum per bladder because of the difficulty in evaluating all lesions in those patients with multiple bladder tumors. Urothelial neoplasms of the urinary bladder may be subdivided into papillary (papillomas, low malignant potential, and papillary carcinoma) and non-papillary (urothelial carcinoma in situ and invasive) categories. In this study, we did not consider the distinct morphological features of the bladder carcinoma with CT or MRI, although further studies should add such a comparison because these morphological features can be important in staging of the bladder carcinoma. 


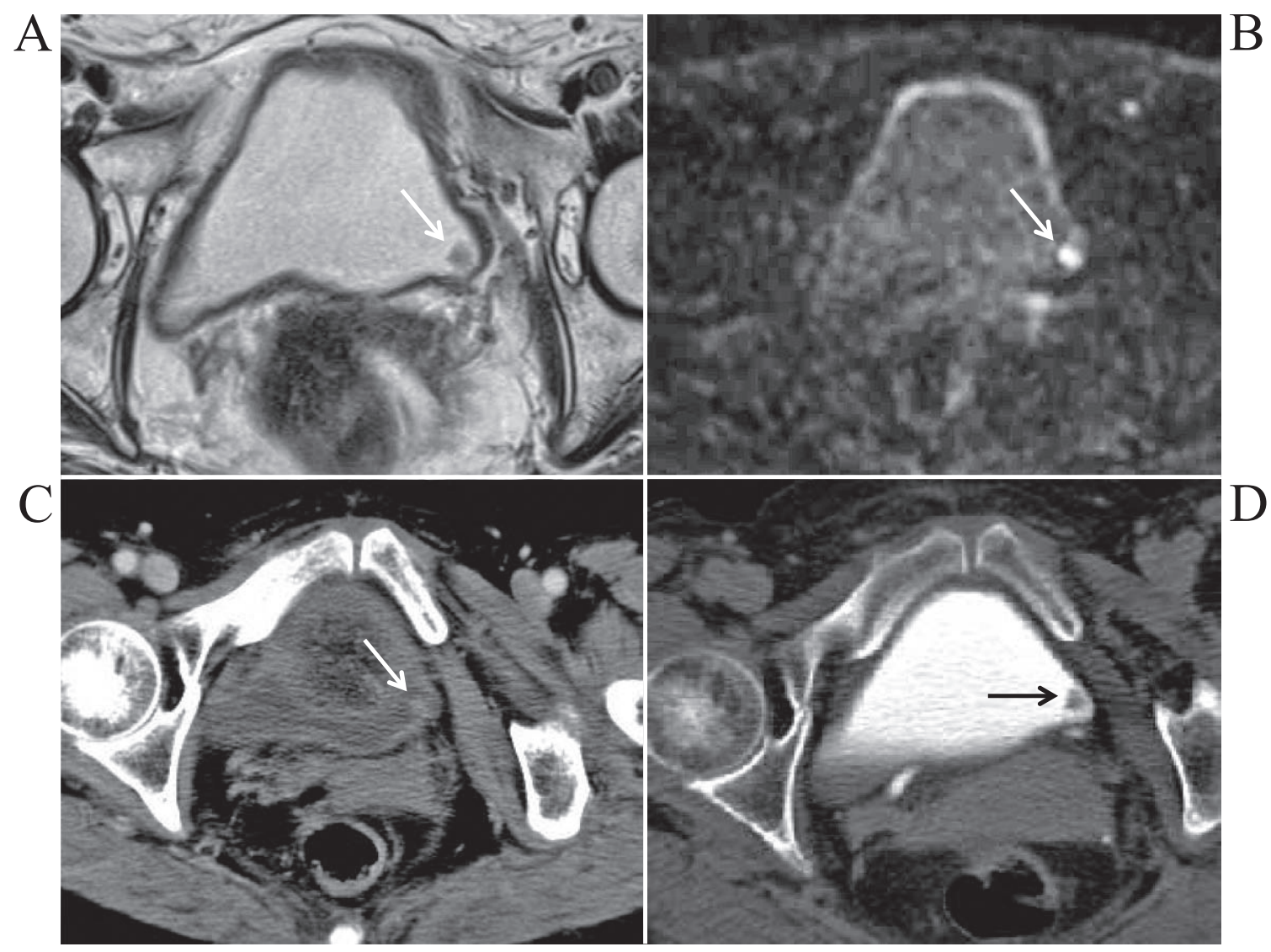

Fig. 1. Bladder cancer on the left posterior wall

A : T2-weighted MR image showing a small tumor in the left posterior wall of the bladder.

B : Diffusion-weighted image demonstrating a high signal tumor (arrow).

C: CT image $80 \mathrm{sec}$ after administration of intravenous contrast agent, showing a small, enhanced nodule in the posterior wall of the bladder.

D : Excretory phase CT showing a small filling defect in the opacified urinary bladder.

A definitive diagnosis of bladder cancer is generally obtained by first confirming the lesion by cystoscopy or transabdominal ultrasonography, followed by histopathological confirmation of tumor tissue excised by a trans-urethral resection of bladder tumor (TURBT) procedure ${ }^{12,17)}$. Trans-abdominal ultrasonography is a non-invasive, convenient testing method that is useful for diagnosing bladder cancer; however, it can be difficult to evaluate the whole bladder by this procedure due to abdominal gas and poor objectivity. Cystoscopy is the most reliable testing method, but it is highly invasive, and not always easy to perform. Cystoscopy should be done when urine cytology is positive, but no bladder tumor is detected on diagnostic imaging, because it is not possible to detect flat lesions or carcinoma in situ on CT or MRI. However, it may be useful for performing CT or MRI prior to cystoscopy because the result gives the urologist gross information of the existence or non-existence of the bladder carcinoma.

CTU has gained wider use recently as a method for urinary tract screening, and high detection rates for bladder cancer are visible in past reports ${ }^{11,12)}$. Among bladder cancer detection rates in the literature, $93 \%$ reported by Turney et $a l^{11)}$ is high compared with our rates of $71.4 \%$ 


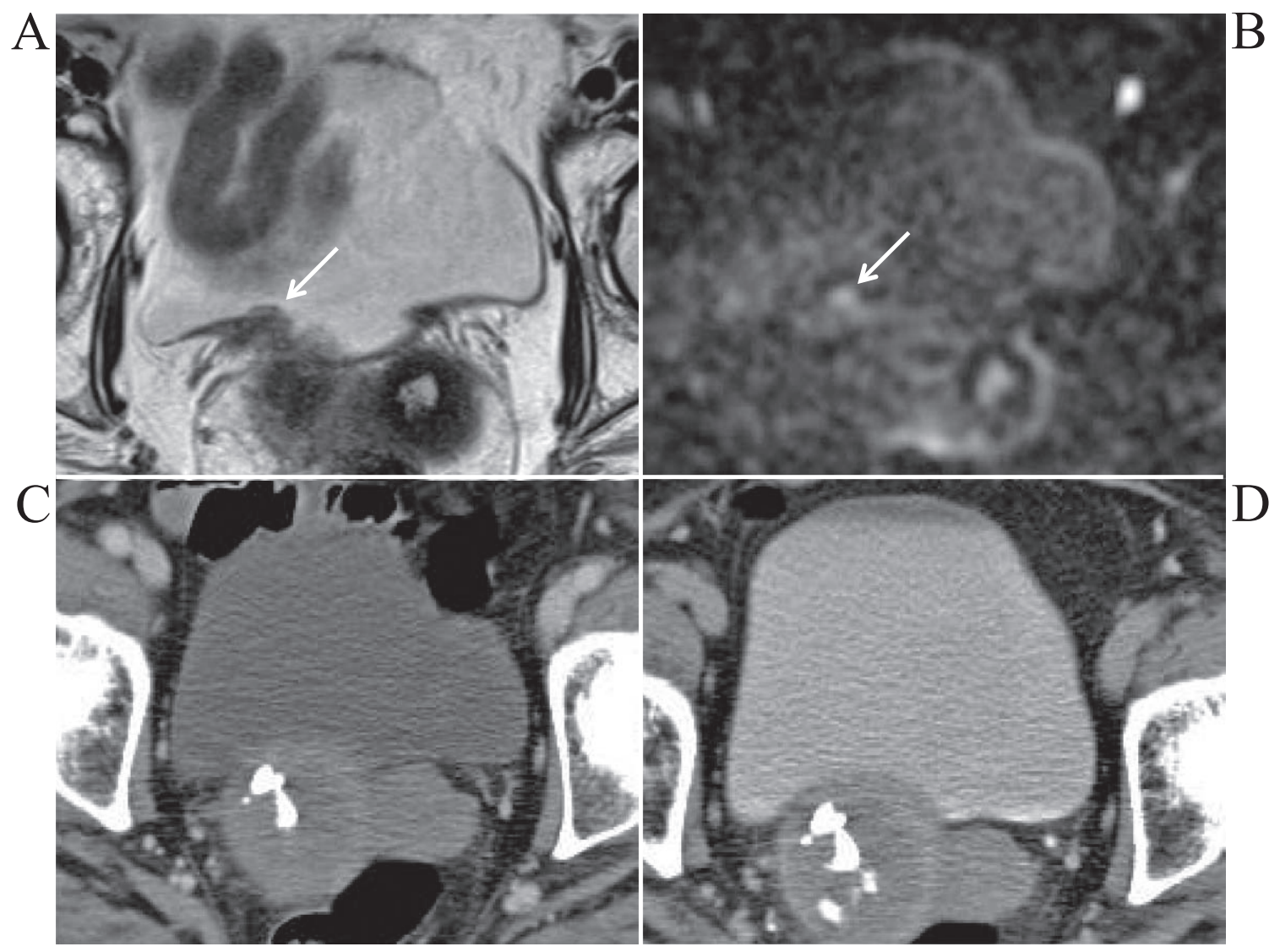

Fig. 2. Bladder cancer in the right posterior wall detected on MRI, but not on CTU

A : T2-weighted MR image showing a small tumor in the right posterior wall of the bladder (arrow).

B : Diffusion-weighted image demonstrating a high signal tumor (arrow).

C: CT image $80 \mathrm{sec}$ after administration of intravenous contrast agent, showing no tumor in the bladder.

D : Excretory phase CT showing no small filling defect in the opacified urinary bladder.

(Reader 1) and $76.9 \%$ (Reader 2), but given that cystoscopy was not performed in our study when no abnormality was present on CTU, it is possible that our values include false negatives. However, our rates support a study by Sadow et $a l^{12)}$ who reported $79 \%$ detection in comparing cystoscopy and CTU findings for all of their patients.

MRI offers excellent tissue contrast, and thus permits satisfactory bladder cancer detection without the use of contrast agents ${ }^{14)}$. In addition, the recently developed diffusionweighted imaging is highly useful for tumor detection generally, and in bladder cancer detection specifically ${ }^{18,19)}$, with reported rates of $98.1 \%{ }^{18)}$ and $89 \%{ }^{19)}$. Compared with our own results of 79.1\% (Reader 1) and 75.1\% (Reader 2), these detection rates are quite high, probably due to differences in tumor size among the studies. Specifically, Abou-El-Ghar et al ${ }^{18)}$ detected a mean bladder tumor size of $29 \mathrm{~mm}$ and Halefoglu ${ }^{19)}$ detected a mean size of $36.6 \mathrm{~mm}$, much larger than the values in the present study. This difference seems particularly affected by the large number of tumors less than $20 \mathrm{~mm}$ in diameter in our cases, for which the mean maximum diameter of tumors was $19.0 \mathrm{~mm}$. 


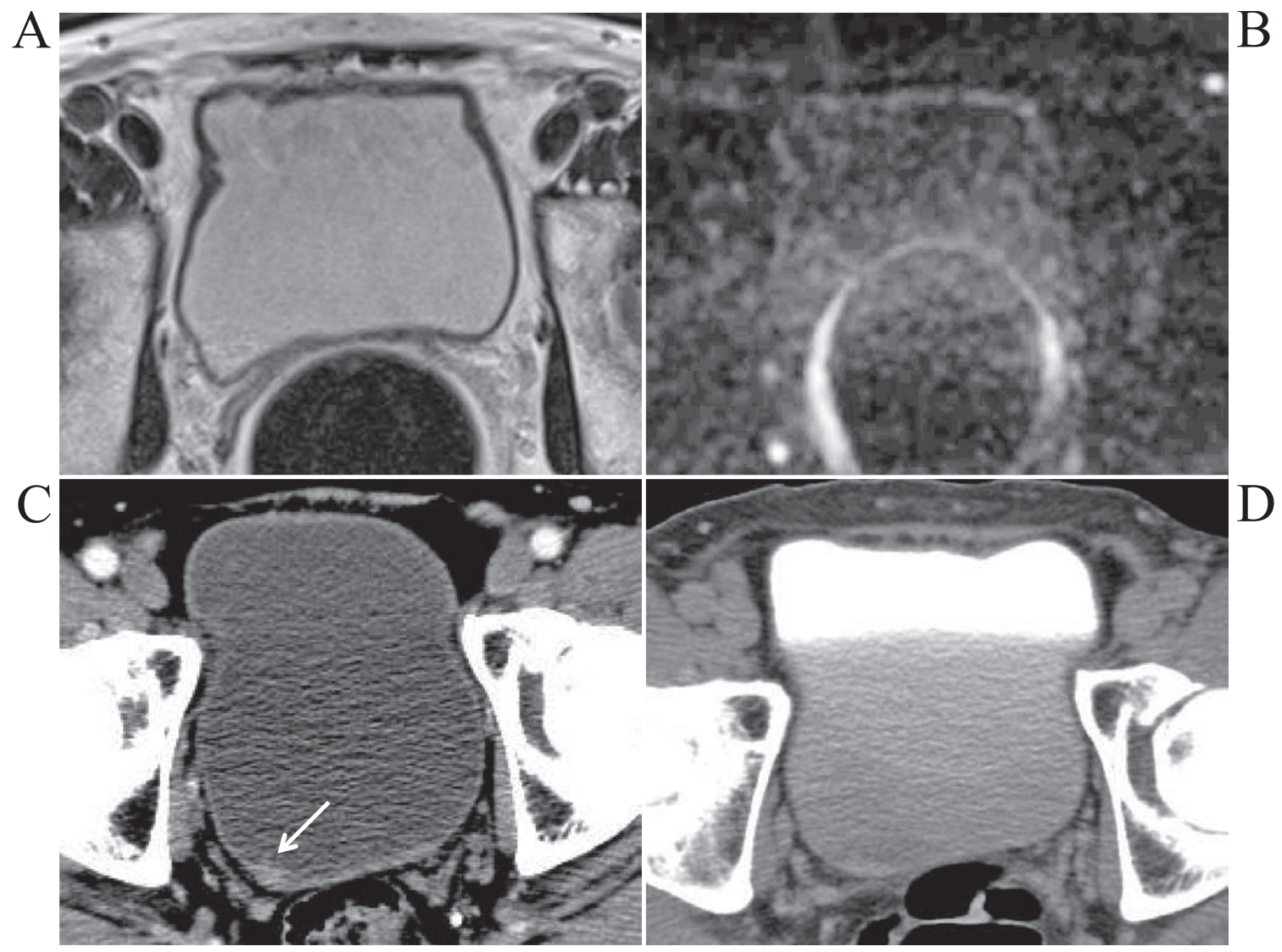

Fig. 3. Bladder cancer on the right posterior wall only seen on enhanced CT

A : T2-weighted MR image showing no tumor in the bladder.

B : Diffusion-weighted image demonstrating no tumor, however the signal may be due to susceptibility artifact from gas in the dilated rectum.

C: CT image $80 \mathrm{sec}$ after administration of intravenous contrast agent, showing a small, enhanced nodule in the right posterior wall of the bladder (arrow).

D : Excretory phase CT showing no definite filling defect in the opacified urinary bladder.

Table 1. CTU vs. MRI for detection of bladder cancer

\begin{tabular}{lcccrr}
\hline \multirow{2}{*}{ Bladder cancer location } & \multicolumn{2}{c}{ Reader 1} & \multicolumn{2}{c}{ Reader 2} \\
& $\mathrm{n}$ & MRI & CTU & MRI & CTU \\
\hline DM & 4 & 2 & 1 & 2 & 2 \\
AW & 3 & 3 & 3 & 3 & 3 \\
PW & 13 & 11 & 8 & 11 & 10 \\
TG & 14 & 11 & 10 & 10 & 10 \\
lt-LW & 29 & 25 & 22 & 24 & 24 \\
rt-LW & 14 & 11 & 10 & 10 & 12 \\
NK & 11 & 8 & 8 & 8 & 8 \\
others & 3 & 1 & 1 & 1 & 1 \\
\hline & 91 & 72 & 65 & 69 & 70 \\
\hline
\end{tabular}

DM : dome TG : trigone NK : neck

AW : anterior wall

PW : posterior wall

lt-LW : left lateral wall rt-LW : right lateral wall 
In our comparison of CTU (renal parenchyma phase and excretory phase) versus noncontrast MRI (T2-weighted and diffusion-weighted), the bladder cancer detection rate by MRI was higher for both Reader 1 and Reader 2, but this difference was not statistically significant. Cystoscopy is the gold standard for bladder cancer detection, but it has been reported that this invasive test can be omitted, patient condition permitting, by conducting CTU instead ${ }^{20-22)}$. Our results suggest that MRI, which requires neither X-ray exposure nor the use of contrast agents, possesses an essentially equivalent detection rate to CTU for bladder cancer. We therefore propose that MRI, with its even-lower invasiveness than CT, could be a superior screening method for bladder cancer.

This research had the following limitations. First, we analyzed only cases of bladder cancer diagnosed by cystoscopy. For this reason, the only measure that was compared between screening methods was detection rate. However, it is clinically difficult to conduct MRI for all patients with suspected bladder cancer based on hematuria, and a prospective study would be necessary to compare CTU and MRI with an adequate measure of specificity. Compared with using cystoscopy, our bladder cancer detection rates for CTU and MRI were certainly not high. Since CTU and MRI were limited to three lesions in cases of multiple bladder cancer, the detection rates would likely drop even lower if the remaining small tumors were included. For MRI to be highly useful as a screening examination for bladder cancer, further progress in MRI equipment as well as improvements in diagnostic techniques are needed.

\section{Conflict of interest disclosure}

The authors have declared no conflict of interest.

\section{References}

1) Itou K, Narumi Y. Disease of the urinary bladder. In Yamashita Y ed. A key to Urologic CT \& MRI. Tokyo: Shujunsha; 2008. pp 170-189. (in Japanese).

2) Jemal A, Siegel R, Xu J, et al. Cancer statistics, 2010. CA Cancer J Clin. 2010;60:277-300. Erratum in : CA Cancer J Clin. 2011;61:133-134.

3) Kirkali Z, Chan T, Manoharan M, et al. Bladder cancer : epidemiology, staging and grading, and diagnosis. Urology. 2005;66(6 Suppl 1):4-34.

4) Van Der Molen AJ, Cowan NC, Mueller-Lisse UG, et al. CT urography: definition, indications, and techniques. A guideline for clinical practice. Eur Radiol. 2008;18:4-17.

5) Lang EK, Macchia RJ, Thomas R, et al. Computerized tomography tailored for the assessment of microscopic hematuria. J Urol. 2002;167:547-554.

6) Lang EK, Macchia RJ, Thomas R, et al. Improved detection of renal pathologic features on multiphasic helical CT compared with IVU in patients presenting with microscopic hematuria. Urology. 2003;61:528-532.

7) Wang LJ, Wong YC, Chuang CK, et al. Diagnostic accuracy of transitional cell carcinoma on multidetector computerized tomography urography in patients with gross hematuria. J Urol. 2009;181:524-531.

8) O’Malley ME, Hahn PF, Yoder IC, et al. Comparison of excretory phase, helical computed tomography with intravenous urography in patients with painless haematuria. Clin Radiol. 2003;58:294-300.

9) Jinzaki M, Matsumoto K, Kikuchi E, et al. Comparison of CT urography and excretory urography in the detection and localization of urothelial carcinoma of the upper urinary tract. AJR Am J Roentgenol. 2011;196:1102-1109. 
10) Kim JK, Park SY, Ahn HJ, et al. Bladder cancer: analysis of multi-detector row helical CT enhancement pattern and accuracy in tumor detection and perivesical staging. Radiology. 2004;231:725-731.

11) Turney BW, Willatt JM, Nixon D, et al. Computed tomography urography for diagnosing bladder cancer. BJU Int. 2006;98:345-348.

12) Sadow CA, Silverman SC, O’Leary MP, et al. Bladder cancer detection with CT urography in an Academic Medical Center. Radiology. 2008;249:195-202.

13) Juri H, Narumi Y. Bladder cancer. Jpn J Diagn Imaging. 2014;34:1306-1316. (in Japanese).

14) Gokan T, Ohbuchi M. MRU (MR urography). In Araki T. MRI of the abdomen. 3rd ed. Tokyo: Medical Science International ; 2014. pp 357-379. (in Japanese).

15) Verma S, Rajesh A, Prasad SR, et al. Urinary bladder cancer: role of MR imaging. Radiographics. 2012;32:371-387.

16) The Japanese Urological Association, The Japanese Society of Pathology, Japan Radiological Society. General Rule for Clinical and Pathological Studies on Renal Pelvic, Ureteral and Bladder Cancer. Tokyo: Kanehara; 2011. (in Japanese).

17) Clark PE, Agarwal N, Biagioli MC, et al. Bladder cancer. J Natl Compr Canc Netw. 2013;11:446-475.

18) Abou-El-Ghar ME, El-Assmy A, Refaie HF, et al. Bladder cancer: diagnosis with diffusion-weighted MR imaging in patients with gross hematuria. Radiology. 2009;251:415-421.

19) Halefoglu AM, Sen EY, Tanriverdi O, et al. Utility of diffusion-weighted MRI in the diagnosis of bladder carcinoma. Clin Imaging. 2013;37:1077-1083.

20) Grossfeld GD, Litwin MS, Wolf JS, et al. Evaluation of asymptomatic microscopic hematuria in adults: the American Urological Association best practice policy--part I: definition, detection, prevalence, and etiology. Urology. 2001;57:599-603.

21) Grossfeld GD, Litwin MS, Wolf JS, et al. Evaluation of asymptomatic microscopic hematuria in adults: the American Urological Association best practice policy--part II: patient evaluation, cytology, voided markers, imaging, cystoscopy, nephrology evaluation, and follow-up. Urology. 2001;57:604-610.

22) Silverman SG, Leyendecker JR, Amis ES Jr. What is the current role of CT urography and MR urography in the evaluation of urinary tract? Radiology. 2009;250:309-323.

[Received November 25, 2015 : Accepted December 14, 2015] 